\title{
4D and In Situ X-ray Microscopy for Studying Damage Evolution in Materials Across Multiple Length Scales
}

\author{
Will Harris ${ }^{1}$, Hrishikesh Bale ${ }^{1}$, Steve Kelly ${ }^{1}$, and Benjamin Hornberger ${ }^{1}$ \\ 1. Carl Zeiss X-ray Microscopy, Pleasanton, CA, USA.
}

Since the emergence of X-ray microscopy (XRM) and tomographic imaging at synchrotron beamlines, continuous improvements in both spatial and temporal resolution have pushed the boundaries of nondestructive 3D characterization. Efficient, semi to fully automated repetitive scanning, along with improved associated image processing and quantification methods, has opened the door to a wealth of new experiments to study evolving microstructures in their native environment or under stimulus, socalled '4D' imaging. In addition, as the frequent development center of advanced X-ray techniques, synchrotron imaging beamlines have also offered an increasing variety of imaging modalities (absorption contrast, phase contrast, diffraction contrast, ptychography, etc).

In recent years, a number of these synchrotron developments have been transferred to analogous laboratory-based instruments; using lab X-ray tubes but incorporating optical elements to achieve resolution and contrast comparable to many synchrotron experiments, and even adopting an increasing variety of imaging modalities. Consequently, as the overall data quality from synchrotron and lab systems has become in many ways comparable, tomography beamlines have increasingly focused on leveraging their unique strength of high X-ray flux to perform dynamic experiments, pushing 3D acquisition times well into the sub-second regime. At this temporal scale, a wide range of dynamic studies capable of capturing rapid microstructural change become possible.

Similarly to the synchrotron community, lab X-ray tomography systems have also benefited from ongoing development of various types of in situ and 4D imaging experiments, albeit at a different time scale, presenting both unique challenges as well as opportunities to the researcher. This work will highlight some of the most recent developments of laboratory-based 4D XRM for materials research, including the potential of static or 'interrupted' in situ operation, and where such longer time-scale operation can be advantageous for example in diffusion, electrochemical, corrosion, ductile deformation, or aging/degradation studies. [1-5]

As part of this discussion the development, operation, and results of a new in situ load stage for nanoscale tomography will also be presented. [6] By filling a 3D characterization gap between microCT (typically on the lengthscale of 1 micron and larger) and TEM (sub-nanometer), nanoscale tomography offers the unique opportunity to probe 3D structures at the tens to hundreds of nanometers scale in a non-destructive manner. To leverage this new scale of investigation, a device has been implemented to apply tension, compression, and nanoindentation loading modes to samples tens to hundreds of microns in size, with the intent of connecting bulk material properties with detailed observations of internal, 3D deformation events occurring at the nano- and micron-scale.

This work will highlight some of the early applications of this technique, including the study of a SiC ceramic matrix composite (CMC) sample subjected to in situ tensile loading and indentation in two separate experiments to investigate the fracture behavior of the material. Due to its strong oxidation resistance and high strength, $\mathrm{SiC}$ is being used in advanced structural applications, but extra processing is required to overcome its inherently brittle behavior. As one route to address this problem, $\mathrm{SiC}$ is 
processed in the form of composites consisting of complex microstructural architectures derived by weaving bundles of fibers, including functional coatings on the fibers and incorporating several strategies to enhance the toughness of bulk composite. The results from the in situ imaging experiment presented here help reveal how cracks initiate and propagate in a $\mathrm{CMC}$ at the nanoscale thereby providing insight into the complex fracture behavior.

\section{References:}

[1] J. Gonzalez et al., Journal of Power Sources, 285 (2015), p. 205.

[2] J. Gelb, D. Finegan, D. Brett, P. Shearing, Journal of Power Sources, 357 (2017), p. 77.

[3] M. Moradllo and T. Ley, Cement and Concrete Composites, 82 (2017), p. 67.

[4] S. A. McDonald et al., Scientific Reports, 7 (2017), 5251.

[5] K. Jespersen and L. Mikkelsen, Data in Brief, 15 (2017), p. 1003.

[6] B. Patterson et al., Experimental Mechanics, 56 (2016), p. 1585.

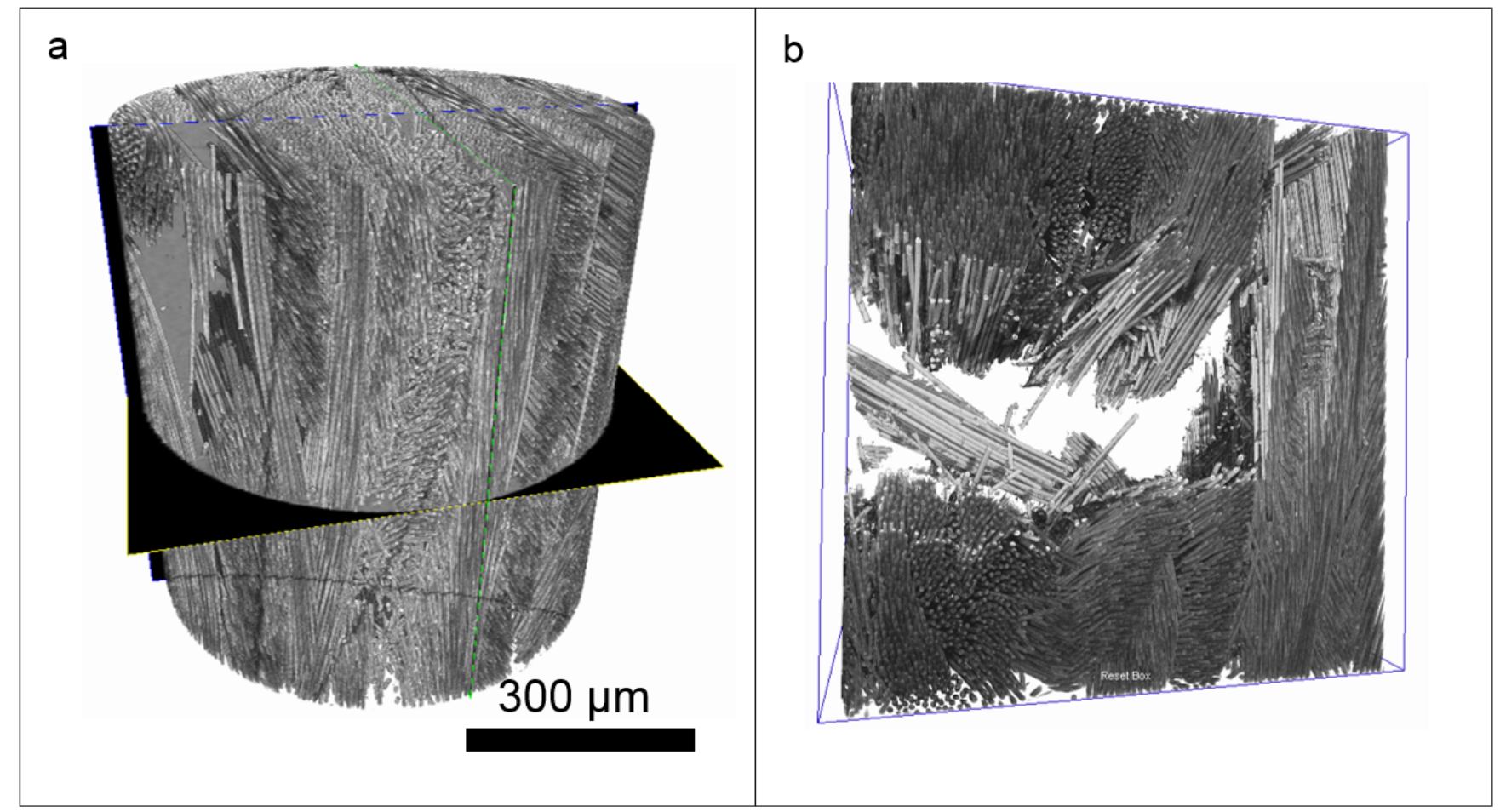

Figure 1. X-ray microscopy scans of a sample of glass fiber reinforced composite, with $2 \times 2 \mathrm{~mm}$ cross section and imaged at $0.9 \mu \mathrm{m}$ voxel size, before (a) and after (b) tensile failure at $180 \mathrm{~N}$ loading. Interrogation of the 3D structure reveals deflection of cracks at ply boundaries. Connection of phenomena at this scale with the finer, single-fiber level scale, will be explored in this paper. 